\title{
Transition to Surgical Practice: The Early Years
}

\author{
Ian M. Paquette, MD, FACS, FASCRS ${ }^{1}$ \\ ${ }^{1}$ Division of Colon and Rectal Surgery, Department of Surgery, \\ University of Cincinnati College of Medicine, Cincinnati, Ohio \\ Clin Colon Rectal Surg 2019;32:457-460.
}

\begin{abstract}
Address for correspondence lan M. Paquette, MD, FACS, FASCRS, Division of Colon and Rectal Surgery, Department of Surgery, University of Cincinnati College of Medicine, 2123 Auburn Avenue \#524, Cincinnati, OH, 45219 (e-mail: ian.paquette@uc.edu).
\end{abstract}

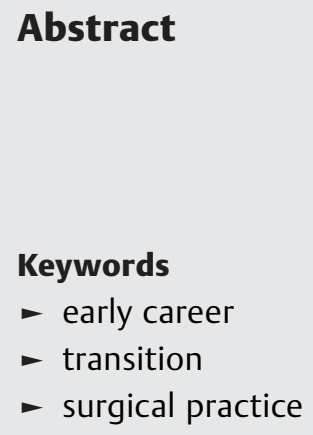

The transition from years of surgical training to independent surgical practice is a time to enjoy the well-earned achievement of completion of training. The early years of clinical practice bring about many challenges to the young surgeon. These include transitioning to a more independent role in the operating room, running an office and understanding the business aspects of medicine, building clinical networks to sustain a clinical practice, and building a professional and academic reputation. This article will discuss aspects that are unique to the early career surgeon. Where possible, I will use the available literature to augment the discussion, whereas, in other areas, I will offer my thoughts from personal experience on the topic.
Since the early days of training in the Halsted era, surgical education has evolved at a rapid pace. In recent times, increasing regulation such as that from the Accreditation Council for Graduate Medical Education (ACGME) has restricted resident duty hours greatly. There is concern among governing bodies such as the American Board of Surgery and the American College of Surgeons (ACS) that residents from general surgery programs may be inadequately prepared for independent surgery practice at the completion of training. ${ }^{1-8}$

Some of the supporting evidence for the lack of preparedness includes a concern that index case numbers have declined as work hours have become restricted. A large study of general surgery residents showed that the total number of operations performed by residents graduating in 2011 actually increased by $21 \%$ compared with residents graduating in $2005 .{ }^{4}$ Though this is encouraging, the proportion of cases considered as index cases by program directors decreased. Of the major operations considered essential in the Surgical Council on Resident Education (SCORE) curriculum, 34\% of these cases were performed a median of less than five times. ${ }^{4}$ Though these changes in duty hours and decreased numbers of index cases do not necessarily impact patient safety, many have called for more flexibility in the surgical training model to maximize the educational value of surgical training. ${ }^{2,9}$

When surveyed directly, surgical residents are concerned as well. A study including more than 4,000 categorical general surgery residents indicated that $27.5 \%$ expressed concerns with being able to perform procedures indepen- dently after graduation. Furthermore, $63.8 \%$ stated that they will choose a subspecialty fellowship to feel ready to operate independently. ${ }^{10}$ This correlates well with a survey of fellowship program directors in the United States. Fellowship directors indicated that $21 \%$ of fellows arrived unprepared for the operating room (OR), 38\% had a lack of ownership of their patients, $30 \%$ could not perform a laparoscopic cholecystectomy, and $66 \%$ were deemed incapable of operating independently for more than 30 minutes. ${ }^{3}$ This discord between residents and program directors deserves further attention.

Lastly, surgical residency and fellowship training may inadequately prepare residents in understanding the business aspects of medicine. A survey of otolaryngology programs revealed that only $8 \%$ of recent graduates rated their business of medicine training as excellent. ${ }^{11}$ Conversely, $75 \%$ of recent graduates rated their business of medicine training as fair or poor. ${ }^{11}$ Similarly, $60 \%$ of recent ophthalmology training program graduates indicated that they were ill prepared to handle the business aspects of practice management. ${ }^{12}$ Though data are limited, there is no reason to believe that a different trend exists in general surgery training.

The sum of these issues has led many to strive to improve general surgery residents' preparedness for practice. Most notably, the ACS and the ACGME convened in 2012 for a 1.5-day summit. ${ }^{13}$ Key recommendations from this conference included an increased focus on the transition to practice, interventions during the chief resident year to better prepare
Issue Theme Surgeon Health; Guest Editor: Jennifer S. Davids, MD, FACS, FASCRS
Copyright (C) 2019 by Thieme Medical Publishers, Inc., 333 Seventh Avenue, New York, NY 10001, USA. Tel: +1(212) 584-4662.
DOI https://doi.org/ 10.1055/s-0039-1693420. ISSN 1531-0043. 
residents for practice, and effective mentorship of junior surgeons as they enter practice. ${ }^{13}$ Based on this, the ACS has developed the "Transition to Practice" program to help assimilate early career surgeons into independent practice. ${ }^{14}$ This is a focused program, where residents leaving a general surgery residency can have a 1 -year paid staff appointment at a participating institution with focused mentorship and education on all aspects of practice management.

The aforementioned collective information is not to discourage young surgeons at the onset of their practice; rather, it should serve to highlight some of the contemporary issues of being ready to enter a surgical career. The knowledge, technical skills, and specialization of surgical techniques are increasing at an astounding pace. The following sections will serve as some practical advice to navigate the transition to an exciting and fulfilling surgical career.

\section{Establish your Clinical Expertise}

The first priority to build both one's own confidence and a clinical reputation in the community is to have outcomes that are nothing short of excellent. As training paradigms have changed, allowing less autonomy during the training years, many early career surgeons are faced with handling surgical cases independently for the first time. This can be made more manageable by planning ahead (reading, studying videos), anticipating critical steps, informing the operative team of equipment needs in advance, working with the OR team to develop preference cards, and obtaining any special equipment in advance. This all goes into developing one's own style, which should be a top priority early on in practice. This is the time to be proactive. I took detailed notes on the steps, exposure, and instrumentation that my mentors used. I made sure that my hospital had all of the correct instrument trays for me and that all preference cards reflected my needs. Handling these details proactively allows one to focus totally on the task at hand in the OR. Additionally, focus on how to be the surgeon in charge of the OR. I have developed different strategies for teaching whether I am with a junior or a senior resident. This is a time to handle more of the cases yourself if needed. Though teaching is important, establishing yourself as a clinical expert with excellent outcomes is perhaps the most important part of a transition to practice.

\section{Work with a Clinical Mentor}

Perhaps the most important asset to the early career surgeon is to have a clinical mentorship strategy. In some instances, this can come from outside of your institution, with former mentors. Ideally, this structure would come from within one's current institution and should cover domains such as surgical skills and judgment, as well as building a clinical practice and assimilating into the culture of the institution. Successful mentorship should meet the mutual expectations of both the mentor and the mentee, and this may include prospective discussion of cases, retrospective feedback regarding operative performance, and assistance with critical intraoperative decision-making, when indicated. This mentorship is most often provided by a more senior surgeon in the department or by a series of mentors such as the senior partners in a division. In addition to helping with prospective thinking about surgical cases, a mentorship strategy is critical in aiding the younger surgeon in dealing with complications as they occur.

\section{Learn the Business Aspects of Surgery}

Though a full discussion of the various models of practice (private, hospital-based, or academic) is beyond the scope of this article, developing more than a basic understanding of billing and coding practices early in one's career is essential. Surgeons need to become as familiar with coding and billing practices as they are with cancer staging algorithms. While undercoding can lead to lower reimbursement for services, overcoding can lead to unintended scrutiny and often penalties from agencies such as the Centers for Medicare \& Medicaid Services. Office encounters will require evaluation and management $(E / M)$ coding. There are specific elements that need to be documented in the medical record depending on the E/M level chosen. Additionally, "modifier codes" can be used if a secondary service such as diagnostic anoscopy is performed on the same visit. Both the $\mathrm{E} / \mathrm{M}$ code and modifier code need to be associated with a diagnosis. Currently, the International Classification of Diseases (ICD-10) system is the most common. Since there are so many ICD-10 codes available, the electronic medical record is often the best source to find the proper code. This is important as the more detail we can provide to reflect the complexity of the patient encounter, the more accurately we can bill. Procedures are billed using current procedural terminology (CPT) codes. It is helpful to reference a CPT codebook when coding for specific procedures. Additionally, be as detailed as possible in dictating operative notes, as additional maneuvers such as splenic flexure mobilization can be coded for. It is important to work closely with your billers and coders to be sure your operative cases are being billed at the appropriate level. A good resource for those interested in learning more on this topic is the ACS. The ACS runs coding and reimbursement workshops, which can be quite helpful in learning the rules of coding effectively. ${ }^{15}$

\section{Become Board Certified}

Another priority of the early career surgeon is to establish one's self as an expert in their respective field. This involves successfully passing both the general surgery and the subspecialty board examinations. Though most young surgeons do pass the board examinations, an alarming trend has emerged with the fail rate for the American Board of Surgery Certifying examination increasing from $16 \%$ in 2006 to $28 \%$ in 2012.5,13 This may often be an effect of the early career surgeon being so focused on improving technical skills, completing a busy fellowship, and developing a clinical practice that issues such as passing the board examination may become a secondary priority. In a specialty field such as 
colon and rectal surgery, an improvement has been seen recently on the written qualifying examination, with only $4 \%$ of candidates failing in 2017 versus 19\% in 2000. However, still, $17 \%$ of candidates failed to pass the oral certifying examination in 2017 (direct communication with the American Board of Colon and Rectal Surgery). It is important to make an honest assessment of one's own abilities and develop a focused study plan to pass the boards. Focused medical knowledge can be improved through a directed study plan, ideally with self-assessment questions to determine areas of strength and weakness. If self-scouting determines that test-taking strategies need to be improved, then signing up for an oral board course or practicing with colleagues and mentors to improve specific test-taking strategies could be beneficial. In any case, the top priority for the early career surgeon needs to be achieving board certification. This will not only help with establishing oneself as an expert in their community but also, in some instances, may be a requirement to achieve academic promotion.

\section{Build a Clinical Practice}

There is no single recommendation for building a clinical practice, and much of this will depend heavily on the local culture. It is, however, important to set expectations. While in residency and fellowship training, there are seemingly endless numbers of patients who need surgery, and the benefit of being a trainee is a constant supply of operative cases. The biggest misconception as many surgeons enter practice is of the types of cases they will be handling at the outset of their career. Unless there are currently no surgeons at your institution with your particular expertise, most early career surgeons will find that the highest proportion of cases early on are the minor bread-and-butter cases. In a colorectal practice, this commonly means seeing mostly patients with anorectal disorders, bowel incontinence, and screening colonoscopy procedures at first. Commonly, in transplant surgery, young surgeons handle far more vascular access cases than solid organ transplants early in their career. This is an opportunity that should be embraced.

It is said that being "available, affable, and able" are the requirements for building a referral practice, and this is certainly true. Make every effort to get to know potential referring doctors, be available at all times to see patients on an urgent basis, and communicate with your patients and families and especially with your referring doctors. Over time, your excellent outcomes will show and you will earn more complex cases a reward for this. Another aspect of building practice involves developing a symbiotic relationship with other specialties. For example, as a young colorectal surgeon, though we are trained to perform colonoscopy, it is often very beneficial to build a trusting relationship with referring gastrointestinal (GI) doctors. A strategy which continues to reap rewards in my own practice is to perform less colonoscopy procedures and instead refer these patients to my GI colleagues. This has allowed them to build trust in my intentions and has led to a robust clinical practice of malignant and benign colorectal diseases and inflammatory bowel disease. Though differing strategies will be needed, depending upon the needs of each community, one major adjustment to make as an early career surgeon is learning how to effectively market oneself and to build a clinical referral network.

Lastly, marketing can be a great opportunity to bring in new patient referrals. Newly practicing surgeons are encouraged to keep an up to date profile on networking sites such as Doximity $^{16}$ and Linkedin ${ }^{17}$ but also to keep their profiles up to date on patient review sites such as Healthgrades ${ }^{18}$ and vitals.com. ${ }^{18}$ It is also helpful to give talks in your community, to give grand rounds to other medical specialties, and, if possible, to appear on television or radio broadcasts to address educational topics and establish yourself as an expert.

\section{Achieve Academic Success}

Achieving a successful academic research career is beyond the scope of this article. However, it is at least worth mentioning that a goal of early career surgeons should be to develop their academic reputation, if this is something that is desired as a career goal. It is now easier than ever to become involved with surgical subspecialty societies, and this is one of the more rewarding aspects of practice. Though there are endless numbers of organizations to become involved with, I would suggest focusing your effort on the one or two that are the most important to you. The American Society of Colon and Rectal Surgeons is likely the best initial society to focus on as an early career surgeon; they have a track record of being inclusive to surgeons from all types of professional practice settings and are always looking for volunteers to serve. Attend the meetings regularly, network with other physicians at the meeting, and volunteer to serve the society. This is most commonly through volunteering for committee service. It is critical to not overextend yourself and take on only what you can complete. To become more academically recognized, the work done for these societies has to be your highest quality work and, above all else, you must adhere to deadlines. Those individuals who continue to show an unwavering dedication to the mission of these societies will enjoy the benefits of increasingly important academic opportunities.

\section{Conclusions}

In summary, the transition to surgical practice is a time of great excitement and anxiety alike. By achieving the basic milestones set out previously, one can start his/her career out on the correct path to a long-term fulfilling endeavor.

Conflict of Interest

None.

\section{References}

1 Yeo HL, Abelson JS, Symer MM, et al. Association of time to attrition in surgical residency with individual resident and programmatic factors. JAMA Surg 2018;153(06):511-517

2 Scally CP, Sandhu G, Magas C, Gauger PG, Minter RM. Investigating the impact of the 2011 ACGME Resident Duty Hour Regulations on 
surgical residency programs: the program director perspective. J Am Coll Surg 2015;221(04):883-9.e1

3 Mattar SG, Alseidi AA, Jones DB, et al. General surgery residency inadequately prepares trainees for fellowship: results of a survey of fellowship program directors. Ann Surg 2013;258(03): 440-449

4 Malangoni MA, Biester TW, Jones AT, Klingensmith ME, Lewis FR Jr. Operative experience of surgery residents: trends and challenges. J Surg Educ 2013;70(06):783-788

5 Lewis FR, Klingensmith ME. Issues in general surgery residency training-2012. Ann Surg 2012;256(04):553-559

6 Crosson FJ, Leu J, Roemer BM, Ross MN. Gaps in residency training should be addressed to better prepare doctors for a twenty-firstcentury delivery system. Health Aff (Millwood) 2011;30(11): 2142-2148

7 Bucholz EM, Sue GR, Yeo H, Roman SA, Bell RH Jr, Sosa JA. Our trainees' confidence: results from a national survey of 4136 US general surgery residents. Arch Surg 2011;146(08):907-914

8 Bell RH Jr, Biester TW, Tabuenca A, et al. Operative experience of residents in US general surgery programs: a gap between expectation and experience. Ann Surg 2009;249(05):719-724

9 Bilimoria KY, Hoyt DB, Lewis F. Perspective of the first trial investigators on accreditation council for graduate medical edu- cation changes in resident work environment and duty hours. JAMA Surg 2017;152(10):903-904

10 Sandhu G, Teman NR, Minter RM. Training autonomous surgeons: more time or faculty development? Ann Surg 2015;261(05):843-845

11 Patel AT, Bohmer RM, Barbour JR, Fried MP. National assessment of business-of-medicine training and its implications for the development of a business-of-medicine curriculum. Laryngoscope 2005;115(01):51-55

12 McDonnell PJ, Kirwan TJ, Brinton GS, et al. Perceptions of recent ophthalmology residency graduates regarding preparation for practice. Ophthalmology 2007;114(02):387-391

13 Sachdeva AK, Flynn TC, Brigham TP, et al; American College of Surgeons (ACS) Division of Education; Accreditation Council for Graduate Medical Education (ACGME). Interventions to address challenges associated with the transition from residency training to independent surgical practice. Surgery 2014;155(05):867-882

14 American College of Surgeons. Mastery in General Surgery Program. https://www.facs.org/education/program/ttp

15 American College of Surgeons. CPT Coding Workshops. https:// www.facs.org/advocacy/practmanagement/workshops

16 Doximity Inc. https://www.doximity.com

17 LinkedIn. https://www.linkedin.com

18 Healthgrades. https://www.healthgrades.com 